\title{
Verhandlungen der Freien Vereinigung der deutschen medizinischen Fachpresse.
}

Am 17. d. M. fand die Generalversammlung der Freien Vereinigung der deutschen medizinischen Fachpresse unter dem Vorsitz ihres Geschäftsführers Hofrat Spatz (München) zu Berlin im Sitzungssaal der Medizinalabteilung des Kultusministeriums statt. Entsprechend der wichtigen Tagesordnung war die Debatte sehr angeregt und eingehend, und nur äußeren Verhältnissen zufolge trat der Schluß schon nach mehr als vierstündiger Dauer ein. Unter den Anwesenden seier namentlich die Herren Professoren Gaffky, Kirchner, Orth, Ewald, E. Kle mperer (Berlin), F.v. Müller (München), Marchand (Leipzig), Wilms (Tübingen), Heubner (Göttingen) aufgeführt, um anzuzeigen, welch lebhaftes Interesse die zur Verhandlung stehenden Gegenstände auch in den Kreisen derjenigen Mitglieder der Vereinigung gefunden hatten, deren redaktionelle Arbeit nur eine sekundäre Rolle in ihrer wissenschaftlichen Tätigkeit spielt. 
Nach dem von Herrn Spatz erstatteten Geschäfts- und Kassenbericht, aus dem der sehr erfreuliche Anstieg der Mitgliederzahl auf rund 150 besondere Erwähnung verdient, kam der erste und bedeutungsvollste Verhandlungsgegenstand, "Reformen; im medizinischen Publikationswesen" zur Diskussion. "Nach, den Referaten der Herren C. Oppenheimer und Posner (Berlin) und auf Grund der Vorschläge, welche die von der vorjährigen Generalversammlung zu diesem Zwecke gewähite Kommission (F. v. Müller, Abderhalden, C. Oppenheimer und Spatz) bekannt gegeben hatten, gelangten folgende Leitsätze zur Annahme:

I. Allgemeines. 1. Die Generalversammlung erkennt an, daß in der medizinischen Publizistik Mißstände vorhanden sind, und erklärt ilıre Bereitwilligkeit, an deren Beseitigung mitzuarbeiten. $\mathrm{Zu}$ diesem Zwecke soll vor allen Dingen dahin gewirkt werden, daß nöglichst alle Herausgeber medizinischer Organe Mitglieder der Freien Vereinigung werden, damit die von ihr gefaßten Beschlüsse eine autoritative Bedeutung besitzen und auf das medizinische Publikationswesen einen inaßgeblichen Einfluß gewinnen.

2. Um der übermäßigen Zersplitterung der medizinischen Literatur zu steuern, hält die Generalversammlung eine möglichste Konzentrierung aller Publikationen auf die Zeitschriften für notwendig. Sie erklärt demzufolge:

3. Die Ausgabe von Festschriften, besonderen Berichten, Jahrbüchern etc. seitens der wissenschaftlichen Institute und Krankenanstalten soll tunlichst beschränkt werden. Die Berücksichtigung von Inauguraldissertationen als Sonderpublikationen ist abzulehnen. Der wesentliche Inhalt solcher Doktorarbeiten soll vom Autor resp. Institutsleiter in Kürze in einer Zeitschrift veröffentlicht werden.

4. In den Originalabhandlungen soll größte Kürze herrschen. Zumal ist die immer wiederholte Anführung zusammengestellter Literaturangaben als überflüssig und schädlich zu verwerfen.

5. Die Zeitschriften sollen möglichst nur solche Arbeiten bringen, die in ihren Rahmen passen.

6. Vorläufige Mitteilungen sollen im allgemeinen nur tatsächliche Feststellungen enthalten. Ihre Veröffentlichung ist nach Möglichkeit einzuschränken.

II. Referatenwesen. 1. Zur Erlangung einer möglichst großen Vollständigkeit auf den Grenzgebieten kann ein Austauschverkehr zwischen den einzelnen Referierorganen stattfinden.

2. Sehr wünschenswert wäre es, wenn die großen Gesellschaften der Spezialfächer den Ausbau des Referierwesens förderten, indem sie geeignete Referatenblätter unterstützten.

Als dritter Punkt der Tagesordnung kam der von J. Schwalbe erstattete Bericht der Kommission zur Bearbeitung der Autoren- und Zeitschriftenliste zur E:ledigung. Mit Genugtuung konnte der Referent feststellen, daß dank den vereinigten Bemühungen der medizinischen Fachpresse und des Verbandes der chemischen Großindustrie die schädliche Arbeit des Soldschreibertums erheblich eingeschränkt worden ist. Diejenigen Arzneimittelfabrikanten, die früher von Aerzten für mehr oder weniger hohe Honorare schwindelhafte Aufsätze über Arzneiwirkungen veröffentlichen ließen, haben auf diese Unterstützung verzichtet, seitdem sie in Erfahrung gebracht haben, daß die Elaborate der Soldschreiber in den der Vereinigung der Fachpresse angehörenden Zeitschriften weder veröffentlicht noch - wenn sie in anderen Journalen erschienen sind - referiert werden.

Nach einem Beschluß der vorjährigen Generalversammlung werden auch die Namen solcher Kollegen, deren Mitarbeit aus anderen Gründen für die Zeitschriften der ,,Vereinigung" unerwünscht sein muß, auf der Liste geführt. Endlich werden auch solche Journale namhaft gemacht, deren redaktionelle Grundsätze zu ethischen Beanstandungen Anlaß geben. - Mit Rücksicht darauf, daß manche der so ,gezeichneten" Autoren und Zeitschriften Büße tun und ,zur Norm zurückkehren", wurde gemäß einem Antrag des Referenten der Beschluß gefaßt, daß von den auf der "Liste" stehenden Autoren und Zeitschriften nach Ablauf eine ${ }_{-}$gewissen Zeit seitens der Kommission diejenigen gestrichen werden, von denen anzunehmen ist, daß sie sich weiterhin den für die Vereinigung der Fachpresse maßgebenden Grundsätzen anpassen werden. - Wichtiger war ein weiterer Antrag des Referenten, der dem Arzneimittelunwesen zu Leibe gehen will. Um namentlich der von manchen Arzneimittelfirmen durch falsche Deklaration ihrer Fabrikate verübten Täuschung der Aerzte entgegenzuwirken, faßte die Generalversammlung den für ihre Mitglieder bindenden BeschluB, daß Inserate und Prospekte von Arzneimitteln und anderen Präparaten, die von der „Kommission" beanstandet werden, in die der Vereinigung angehörenden Zeitschriften nicht mehr aufgenommen werden. (Selbstverständlich werden auch einpfehlende Aufsätze über solche beanstandeten Fabrikate von der Publikation ausgeschlossen werden.)

Ueber die von Herrn W. Heubner (Göttingen) in den Therapeutischen Monatsheften 1910, No. 4 und 6 aufgestellte Forderung, die Abgabe ron Sonderdrucken zu Propagandazwecken für unzulässig zu erklären, berichtete Herr Lennhoff. In den Schlußsätzen seines ge- druckt vorliegenden Referates gelangt er zu einer Ablehnung des Heubnerschen Antrages. Nach eingehender Debatte, an der isich auch die vom Vorstand eingeladenen Verlagsbuchhändler Herren Meiner (Leipzig) und Springer (Berlin) beteiligten, wird beschlossen, mit Rück sicht auf verschiedene rechtliche Unklarheiten die Frage als noch nicht spruchreif anzusehen und auf die Tagesordnung der nächstjährigen Generalversammlung zu setzen. Zur Bearbeitung des Gegenstandes wird eine Kommission, bestehend aus den Herren $\mathrm{Heu}$ bner, Lennlı off, Kantor, Kraft und Springer eingesetzt.

Herr Posner machte Mitteilung von den Beschlüssen der letzten (gelegentlich des Budapester Internationalen medizinischen Kongresses abgehaltenen) Versammlung der Internationalen Vereinigung der medizinischen Fachpresse: 1. Betreffs der Frage der „ReklameArtikel" hat die Generalversammlung der Association internationale de la Presse médicale eine Resolution angenommen, in der die Aufnalime und der Abdruck solcher Artikel ,untersagt" wird, außerdem aber eine Internationale Kommission zum Studium dieser Frage und namentlich zur Definition der Bezeichnung ,Reklameartikel" eingesetzt (Antrag Neustätter). Die Generalversammlung ist mit dieser Kominissionsberatung einverstanden. 2. Die Budapester Versainmlung sprach sich für Einsetzung einer Permanenten Internationalen Kommission für die großen Kongresse aus. Solche ist in Budapest gewählt; †hr gehört der Vorsitzende der Internationalen Vereinigung (zurzeit Lucas-Championnière) de jure an; Deutsch land ist durch Waldeyer und Posner vertreten. 3. Rücksichtlich der vielfach gewünschten ,Zusammenfassungen" anı SchluB der Arbeiten hat die Versammlung in Budapest beschlossen, daß solche bei Artikeln, die in einer der wenig verbreiteten Sprachen erscheinen - also etwa russisch, magyarisch etc. - in einer der drei Weltsprachen beigegeben werden sollten, in übrigen aber freie Hand gelassen werden solite. 4. In der Frage der gleichzeitigen Publikation einer Arbeit in mehreren Sprachen hat der Internationale Kongre $\mathbb{S}$ solches für zulässig erklärt, falls die Arbeit in einer ,wenig verbreiteten Sprache“ erschienen sei. Die Generalversammlung stimmt dem zu unter der Voraussetzung, daß dann jedesmal auf die gleichzeitig erscheinende anderweitige Publikation ausdrücklich hingewiesen wird. 5. Zukünftig sollen alle der Association internationale angehörigen Journale die Bezeichnung ,Journal affilié à l'Association internationale de la Presse médicale" tragen. - Die Generalversammlung behält sich vor, diese Frage auf ihrer nächsten Versammlung inı Zusammenhang mit der Frage, ob auch die Zugehörigkeit zur Deutschen Vereinigung kenntlich gemacht werden solle, eingehend zu beraten.

Auf Antrag des Herrn Joachim (Berlin) wurde beschlossen, auf der nächsten Generalversammlung die Beratung ,einer einheitlichen Zitiermethode in medizinischen Publikationen" anzusetzen; Herr Joachim wurde mit der Ausarbeitung von Vorschlägen betraut. Angenommen wurde schließlich ein Antrag des Herrn Schnirer (Wien), der sich gegen Mißstände wendet, die namentlich in jüngster Zeit bei den Publikationen über Erfolge des Ehrlichschen Mittels hervorgetreten sind: „Die Freie Vereinigung der medizinischen Fachpresse erklärt es für unzulässig, Artikel von Autoren anzunehmen, die den Inhalt ihrer einem Fachblatte eingereichten Arbeiten vor deren Erscheinen der Tagespresse mitteilen."

Der vorjährige Vorstand wurde durch Akklamation wiede gewähit. An die Sitzung schloß sich in Monopol-Hotel ein gemeinsames Abendessen.

Die Generalversammlung des 17. September hat einen weiteren erfreulichen Beweis dafür geliefert, daß die Freie Vereinigung der inedizinischen Fachpresse rüstig vorwärts schreitet in der Erfüllung der Auf gaben, die sie zum Besten der deutschen medizinischen Literatur und der ärztlichen Standesinteressen zu lösen hat. J. S. 